\title{
BMJ Open Open-labelled randomised controlled trial of 12 hours versus 24 hours modified Pritchard regimen in the management of eclampsia and pre- eclampsia in Ghana (MOPEP Study): study protocol
}

Titus Beyuo, ${ }^{1}$ Emma Lawrence (I) ,2 Elizabeth S Langen, ${ }^{2}$ Samuel A Oppong ${ }^{1}$

To cite: Beyuo T, Lawrence $\mathrm{E}$, Langen ES, et al. Openlabelled randomised controlled trial of 12 hours versus 24 hours modified Pritchard regimen in the management of eclampsia and pre-eclampsia in Ghana (MOPEP Study): study protocol. BMJ Open 2019;9:e032799. doi:10.1136/ bmjopen-2019-032799

- Prepublication history for this paper is available online To view these files, please visit the journal online (http://dx.doi. org/10.1136/bmjopen-2019032799).

Received 07 July 2019 Revised 12 September 2019 Accepted 24 September 2019

Check for updates

(c) Author(s) (or their employer(s)) 2019. Re-use permitted under CC BY-NC. No commercial re-use. See rights and permissions. Published by BMJ.

${ }^{1}$ Obstetrics and Gynaecology, University of Ghana School of Medicine and Dentistry, Accra, Ghana

${ }^{2}$ Obstetrics and Gynecology, University of Michigan, Ann Arbor, Michigan, USA

Correspondence to

Dr Emma Lawrence; emmarl@med.umich.edu

\section{ABSTRACT}

Introduction Hypertensive disorders of pregnancy continue to be a major contributor to maternal and perinatal morbidity and mortality. Magnesium sulfate therapy is the standard of care for seizure prophylaxis and treatment for pre-eclampsia and eclampsia respectively, despite wide disparities in dosing regimens and routes of administration. This study compares the clinical efficacy of magnesium sulfate in the reduction of seizure occurrence or recurrence with the 12 hours versus 24 hours modified Pritchard regimens in the management of severe preeclampsia and eclampsia.

Methods and analysis This study is an open labelled randomised controlled trial. The study participants are patients admitted to the Korle Bu Teaching Hospital (KBTH) in Accra, Ghana with a diagnosis of antepartum, intrapartum or postpartum eclampsia or pre-eclampsia with severe features. All study participants will be administered a loading dose of magnesium sulfate, followed by maintenance dosing. Participants in the control group will receive magnesium sulfate for 24 hours after diagnosis, while those in the treatment group will receive magnesium sulfate for 12 hours after diagnosis. The primary outcome of this study is the occurrence of a seizure any time after the completion of treatment in the assigned group. Secondary outcome measures include maternal health outcomes, magnesium sulfate toxicities and fetal health outcomes. Data collection was started in October 2018 with a target enrolment of 1245 participants with severe pre-eclampsia and 844 participants with eclampsia with a projected study period of 2-3 years.

Ethics and dissemination Ethical approval was obtained from the KBTH Institutional Review Board (IRB) in Ghana. University of Michigan involvement is limited to protocol development and statistical analysis of de-identified data, and has been granted a Not Regulated Determination by the University of Michigan IRB. Results of the study will be shared at clinical forums at the $\mathrm{KBTH}$ and will be submitted for publication in an international peer-reviewed journal.

\section{Strengths and limitations of this study}

- Large randomised controlled trial that addresses duration of administration of magnesium sulfate, which fills an important gap in the literature and has significant implications for management of patients with eclampsia and pre-eclampsia with severe features.

- The study is taking place in a low resource setting with a high incidence of pre-eclampsia and eclampsia and advanced disease at presentation.

- Data collection in a developing country setting with no electronic medical record may contribute to missing information and become a study limitation.

- Lack of blinding of patients and providers to group allocation is a study limitation, however, primary outcome (occurrence of seizure) and secondary outcomes (maternal: clinical toxicities and complications; neonatal: birth outcomes) are objective measures.

Trial registration number Pan African Clinical Trial Registry through the South African Medical Research Council (PACTR201811515303983).

\section{INTRODUCTION}

Hypertensive disorders complicate $10 \%$ of pregnancies worldwide ${ }^{12}$ and pre-eclampsia occurs in $2 \%-8 \%$ of all pregnancies globally. ${ }^{1}$ In developing countries, hypertensive disorders account for $10 \%-15 \%$ of maternal deaths. ${ }^{2}$ In Ghana, the incidence of hypertensive disorders in pregnancy has been estimated from a hospital based study as $7.6 \% .^{34}$ Eclampsia contributes significantly to maternal mortality in developing countries. In Ghana, a case fatality of $3.9 \%$ has been reported, and institutional reports from two major tertiary-level hospitals suggest 
hypertensive disorders have overtaken haemorrhage as the leading cause of maternal mortality. ${ }^{3}$

While pre-eclampsia may not be preventable, the risk of eclampsia can be significantly reduced with good obstetric management. ${ }^{56}$ Magnesium sulfate has been used for decades for the management of eclampsia and pre-eclampsia and is the anticonvulsant of choice for the management and prevention of eclamptic seizures. ${ }^{78}$ Good clinical efficacy has been reported with the use of magnesium sulfate in the management of pre-eclampsia despite wide disparities the dosing regimens and routes of administration. ${ }^{57-12}$

There is lack of consensus in the existing literature about optimal duration and dosing of magnesium sulfate, as well as optimal therapeutic window. Therapeutic failures have been reported for doses considered both high and low. ${ }^{79-12}$ The narrow therapeutic window widely used in literature is challenged by pharmacokinetic studies which demonstrate that accepted international regimens sometimes fail to reach the minimum therapeutic threshold yet are associated with clinical efficacy. ${ }^{73}{ }^{714}$ The duration of treatment has also been questioned, and a wide range of different regimens are currently used in clinical practice. Protocols such as the Sokoto (ultra short) protocol demonstrated that just the standard loading dose of the Pritchard regimen may be sufficient. ${ }^{11}$

In Korle $\mathrm{Bu}$ Teaching Hospital (KBTH) and other centres in Ghana, many patients do not complete the 24 hours maintenance course due to the poor compliance and discomfort associated with the repeated injections, side effects and sometimes financial constraints; yet our observational experience has not demonstrated an increase in disease progression in this cohort of patients. Perhaps, this is secondary to the efficacy demonstrated in shorter regimens like the 12 hours maintenance and ultra-short protocol. ${ }^{5}$ The cost to the individual and the healthcare system associated with the 24 hours maintenance course is significant. The determination of the minimum effective duration of treatment with proven clinical efficacy, minimum side effect profile and toxicity is likely to improve compliance, reduce waste and conserve resources in developing countries.

\section{Problem statement}

Maternal morbidity and mortality resulting from pre-eclampsia and eclampsia remain high in many middle to low income countries. The case fatality of eclampsia is unacceptably high despite known interventions to reduce the progression to eclampsia. The current dosing regimens are at best empirical with several different regimens being used in clinical practice.

Previous studies comparing clinical efficacy of dosing regimens involved relatively small sample sizes and were not powered to detect clinical differences in toxicity and tolerability. Two small studies have compared outcomes using 12 hours versus 24 hours of magnesium sulfate using an intravenous infusion regimen. ${ }^{5}$ However, there are no studies that compare 12 hours versus
24 hours of magnesium sulfate using an intramuscular regimen, which is a common method of administration in developing countries, particularly in Africa. ${ }^{15}$ Finally, the incidence of the various side effects or toxicities of magnesium sulfate with the Pritchard regimen has not been studied in the Ghanaian population, despite years of utilization of this regimen.

\section{Justification}

Magnesium sulfate remains the mainstay of treatment prevention of eclamptic seizures in patients with pre-eclampsia and the prevention of recurrent seizures in patients with eclampsia. The clinical efficacies reported with varying durations and routes of administration necessitates further research to determine the optimum effective duration with demonstrable clinical efficacy and low toxicity which will be cost-efficient to the healthcare system, especially in low-resource countries.

Our study therefore seeks to investigate and compare clinical response between the 12 hours versus 24 hours modified Pritchard regimens. A significant reduction in cost is expected if the reduced duration of treatment proposed by this trial is adopted and there is a need to subject it to a rigorous clinical trial to assure maternal safety.

\section{Aim and objective}

Aim

To compare the 12 hours versus 24 hours modified Pritchard regimens of magnesium sulfate in the management of eclampsia and pre-eclampsia with severe features.

\section{Objectives}

1. To compare the clinical efficacy of the 12 hours versus 24 hours modified Pritchard regimens in the reduction of seizure occurrence in women with eclampsia and pre-eclampsia with severe features.

2. To compare the side effect profile and toxicity of the 12 hours versus 24 hours modified Pritchard regimens in the management of women with eclampsia and pre-eclampsia with severe features.

3 . To compare the neonatal health outcomes following treatment with 12 hours versus 24 hours modified Pritchard regimens in women with eclampsia and pre-eclampsia with severe features.

\section{Hypotheses}

$\mathrm{H}_{0}$ : There is no difference in clinical efficacy of magnesium sulfate between the 12 hours versus 24 hours modified Pritchard regimens.

$\mathrm{H}_{1}$ : There is a difference in clinical efficacy of magnesium sulfate between the 12 hours versus 24 hours modified Pritchard regimens.

\section{METHODS AND ANALYSIS}

\section{Study design}

An open-labelled randomised controlled trial in which the study arm will receive 12 hours of maintenance doses 
of magnesium sulfate and the control arm will receive 24 hours of maintenance doses of magnesium sulfate. Participants will be enrolled into study groups by simple randomisation using a computerised random number generation table.

\section{Study site}

The KBTH is the country's premier teaching hospital and serves as a national referral hospital for the southern half of the country. It is a tertiary hospital with a bed capacity of 2000 and an average daily outpatient department attendance of 1500 patients. The maternity unit conducts about 9500 deliveries per year. Current standard of care at KBTH is a modified Pritchard regimen of magnesium sulfate, with administration of a loading dose followed by 24 hours of maintenance dosing, starting at the time of diagnosis of eclampsia or pre-eclampsia with severe features.

\section{Study population}

Accra is a cosmopolitan city with people of varied ethnic backgrounds and social status. It is expected that the sample will represent this diversity and be reflective of the nation. Participants will be patients receiving their prenatal care at KBTH, as well as referral cases from periurban and rural communities around Accra.

\section{Inclusion criteria}

1. Clinical diagnosis of antepartum, intrapartum, or postpartum eclampsia based on:

a. Elevated blood pressures of 140/90 mm Hg or higher on two occasions at least 4 hours apart after 20 weeks of pregnancy and

b. Seizures not attributed to other causes.

2. Clinical diagnosis of pre-eclampsia with severe features (definitions per ACOG Hypertension in Pregnancy Guidelines ${ }^{16}$ ) based on:

a. Elevated blood pressures of $160 / 110 \mathrm{~mm} \mathrm{Hg}$ or higher on two occasions at least 4 hours apart after 20 weeks of pregnancy, with proteinuria or presence of laboratory values or clinical symptoms indicating end-organ dysfunction or

b. Elevated blood pressures of 140/90 mm Hg or higher on two occasions at least 4 hours apart after 20 weeks of pregnancy, with presence of laboratory values or clinical symptoms indicating end-organ dysfunction.

3. Patients who receive loading dose of $\mathrm{MgSO}_{4}$ at referral clinics prior to referral will be eligible for randomisation if they otherwise meet the inclusion criteria.

4. Age 18 years or older.

\section{Exclusion criteria}

1. Eclampsia complicated by acute renal failure, HELLP syndrome (hemolysis, elevated liver enzymes and low platelet count) or pulmonary oedema.

2. Comorbid maternal diagnosis of renal disease and/or seizure disorder.
3. Contraindication to $\mathrm{MgSO}_{4}$ (eg, drug hypersensitivity, myasthenia gravis, anuria or oliguria).

4. Prior intake of any other anticonvulsant.

5. Prior exposure within 72 hours to magnesium sulfate which was not a component of the study regimens

6. Refusal to give consent or unable to give consent (eg, unconscious).

7. Age 17 or younger.

\section{Sample size estimation}

An occurrence of a seizure is the primary outcome. In women with eclampsia, this will be a recurrent seizure and in women with pre-eclampsia with severe features, this will be their initial seizure.

Regarding eclampsia, we assume that the recurrent seizure rate of women receiving 12 hours of magnesium will be halfway between the seizure rate of women receiving 24 hours of magnesium $(13.2 \%)$ and women receiving no magnesium $(27.9 \%)$ at $20.6 \% .{ }^{17}$ To detect a difference between $20.6 \%$ recurrent seizure in the 12 hours group and $13.2 \%$ recurrent seizure in the 24 hours group, a total sample size of 804 (402 in each group) is needed, assuming a two-sided test, $5 \%$ significance level and $80 \%$ statistical power.

Regarding pre-eclampsia, in the subgroup analysis of the Magpie Trial, women with severe pre-eclampsia have a $1.1 \%$ rate of seizure if treated with 24 hours of magnesium. ${ }^{10}$ In our current study, with a high-risk population, we anticipate a $6 \%$ rate of seizures in women who receive no magnesium based on study site department data. In absence of data from the literature, we assume that the seizure rate of women receiving 12 hours of magnesium will be halfway between the seizure rate of women receiving 24 hours of magnesium $(1.1 \%)$ and women receiving no magnesium $(6 \%)$ at $3.55 \%$. To detect a difference between $3.55 \%$ seizures in the 12 hours group and $1.1 \%$ seizures in the 24 hours group, a total sample size of 1186 (593 in each group) is needed, assuming a two-sided test, 5\% significance level and $80 \%$ statistical power.

Assuming 5\% lost to follow-up and intervention discontinuation, 844 women with eclampsia (422 in each group) and 1245 women with severe pre-eclampsia (623 in each group) were recruited. See figure 1 for Consolidated Standards of Reporting Trials (CONSORT) flow chart.

\section{Study duration}

Conceptualisation and securing of appropriate Institutional Review Board (IRB) approvals and funding and organisation of trial materials lasted 18 months. Trial recruitment and follow-up is estimated to last 2-3 years starting October 2018.

\section{Protocol}

All women admitted to the maternity ward of KBTH as an antepartum, labouring or postpartum patient will be assessed by the on-call obstetric resident according to the standard clinical practice. Based on initial history and 


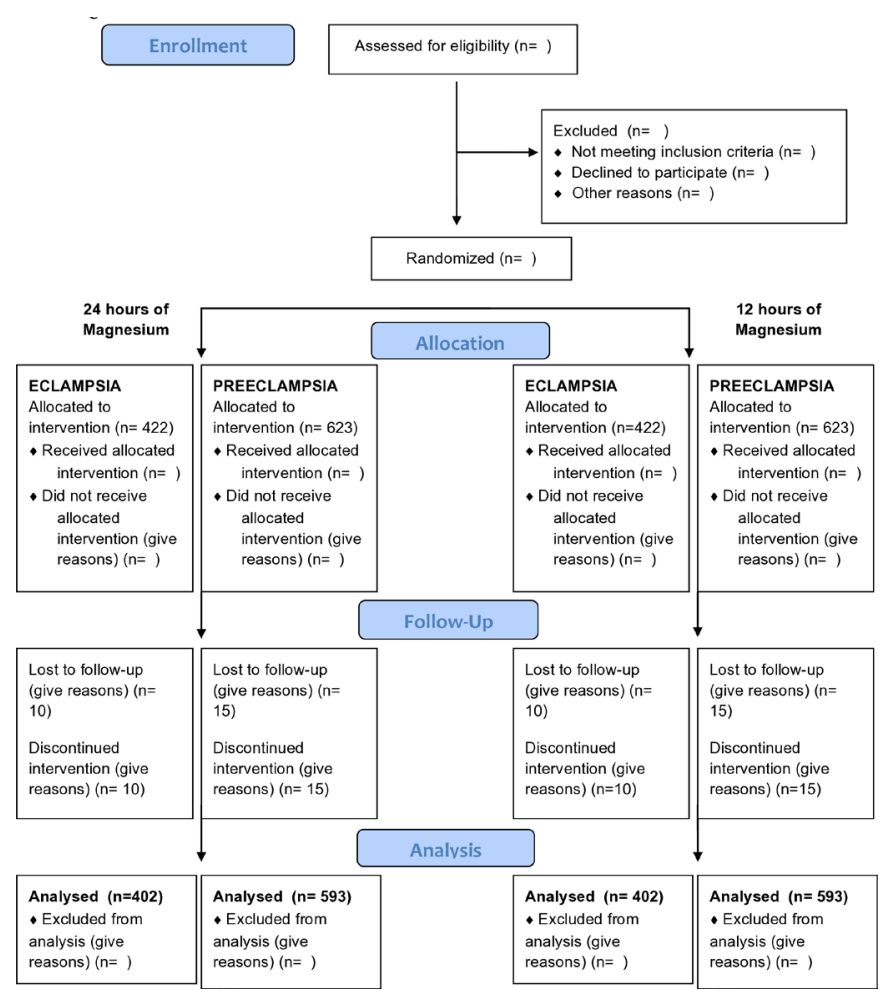

Figure 1 Recruitment and enrolment. CONSORT flow diagram outlining participant flow through each stage of the randomised controlled trial (intervention allocation, follow-up and data analysis). CONSORT, Consolidated Standards of Reporting Trials.

physical examination, vitals signs and laboratory values, clinical diagnoses will be made by the on-call obstetric team. If a patient has a clinical diagnosis of eclampsia or pre-eclampsia with severe features, she will be identified as a potential study participant. The on-call resident will review study inclusion and exclusion criteria. If a woman meets inclusion criteria, a research assistant will be notified to confirm inclusion/exclusion criteria and obtain informed consent (see consent form in suppplementary file).

Participants will be enrolled into study groups by simple randomisation using a computerised random number generation table (Random Number Generator V.3.0.56 for Mac). Using the table of random sequence of numbers ( 1 vs 2 ), sequentially numbered printed data collection forms will be labelled ( $1=$ control group; $2=$ treatment group) by researcher TB. After a participant is recruited and consented by the research assistant, the research assistant will be given the next numbered data collection form indicating the randomisation group. The research assistants are not involved in the randomisation process and do not have access or knowledge of the next treatment allocation. A sticker will be placed on the participants' chart to notify her clinical team of her participation and group assignment.

The research assistant will commence data collection by extracting relevant demographic and clinical data, such as medical history, past obstetric history and history of the index pregnancy from the participant's antenatal record and supplemented by direct interview of participants. During the hospitalisation, we will prospectively document events such as timing of magnesium sulfate doses, timing of any seizures, mode and timing of delivery, maternal complications and magnesium sulfate toxicities. Neonatal information will be collected, including gestational age at delivery, birth weight, outcome of delivery (live birth vs stillbirth), neonatal intensive care unit (NICU) admission, APGAR score at 1 and 5 min and status at discharge (alive vs dead).

According to the standard protocol at KBTH, all participants will have a detailed history and physical examination done at the time of admission. Complete blood counts, clotting profile, liver and renal function tests and urine protein measurements will be performed. Strict fluid input and output monitoring will be done and recorded on the study fluids chart. All patients will have urethral catheterisation retained for continuous bladder drainage until the last dose of the magnesium sulfate. All participants will be monitored for the entire duration of $\mathrm{MgSO}_{4}$ treatment by the on-call obstetrics team at least every 4 hours for blood pressure, patellar reflexes, respiratory rate, urine output and occurrence of seizures. After completion of the $\mathrm{MgSO}_{4}$ injections, patients will have their blood pressure monitored every 4 hours until normalisation of blood pressure, and subsequent discharge.

Apart from the duration of magnesium sulfate administration, all other clinical management of study participants will be carried out by the attending on-call obstetrician in accordance with the standard institutional care protocol at KBTH. This includes administration of antihypertensives, decision on timing and mode of delivery, induction of labour and initiation and interpretation of fetal monitoring.

\section{Main intervention: duration of magnesium sulfate}

1. Loading dose: all study participants will receive a loading dose of $4 \mathrm{~g}$ of intravenous $\mathrm{MgSO}_{4}$ and $10 \mathrm{mg}$ intramuscular $\mathrm{MgSO}_{4}$ ( $5 \mathrm{~g}$ in each buttock) given at the time of antepartum, intrapartum or postpartum diagnosis of eclampsia or pre-eclampsia with severe features.

2. Maintenance doses:

a. Treatment group (12 hours, modified Pritchard): $5 \mathrm{~g} \mathrm{MgSO}_{4}$ intramuscular every 4 hours for a total of three doses over 12 hours starting at the time of diagnosis of eclampsia or pre-eclampsia with severe features.

b. Control group (24hours, modified Pritchard): $5 \mathrm{~g}$ $\mathrm{MgSO}_{4}$ intramuscular every 4 hours for a total of six doses over 24 hours starting at the time of diagnosis of eclampsia or pre-eclampsia with severe features.

All doses of magnesium sulfate will be prepared and verified by the KBTH in accordance with standard hospital protocols. The loading doses will be constituted as $8 \mathrm{~mL}$ of $50 \%$ solution diluted with $12 \mathrm{~mL}$ of sterile saline to obtain $20 \mathrm{~mL}$ of $4 \mathrm{~g} \mathrm{MgSO}_{4}$, to be administered 
slowly over 15-20 min. Maintenance doses will be constituted as $10 \mathrm{~mL}$ of $50 \% \mathrm{MgSO}_{4}$ solution to obtain $5 \mathrm{~g}$ of $\mathrm{MgSO}_{4}$, to be given as deep intramuscular injection. All doses of magnesium sulfate will be administered by clinical hospital nursing staff in accordance with standard hospital protocols.

\section{Treatment failure}

The occurrence of a seizure after completion of the third maintenance doses (MD3) and sixth maintenance doses (MD6) for the study and control groups, respectively will be regarded as a treatment failure. These patients will have the 24 hours modified Pritchard protocol restarted and completed if clinical assessment permits further magnsium sulfate therapy. After treatment failure, the management of further magnesium Sulfate is at the clinical discretion of the attending on-call obstetrician.

The occurrence of clinical evidence of toxicity (absent tendon reflexes, respiratory depression, coma) after initiation of maintenance doses that necessitate discontinuation of further maintenance doses will also be regarded as a treatment failure. In the case of $\mathrm{MgSO}_{4}$ toxicities, the plan of management will be to stop further administration of $\mathrm{MgSO}_{4}$ and inject $1 \mathrm{~g}$ of calcium gluconate $(10 \mathrm{~mL}$ of $10 \%$ solution) intravenously. The decision to reinitiate magnesium Ssulfate is at the clinical discretion of the attending on-call obstetrician.

\section{Outcome measures}

The primary outcome of this study will be the clinical efficacy of the magnesium sulfate regimen, defined as the absence of a seizure any time after the completion of the MD3 until discharge in the treatment (12 hours) group and after the completion of the MD6 until discharge in the control (24hours) group.

Secondary outcome measures include:

Maternal:

1. The interval from initiation of treatment to the development of diuresis (diuresis will be defined as urine output $>400 \mathrm{~mL} / 4$ hours) .

2. Clinical evidence of toxicity (absent tendon reflexes, Respiratory rate (RR) $<16 \mathrm{cpm}$, coma).

3. Side effects of magnesium (nausea/emesis, muscle weakness, absent or reduced reflexes, respiratory depression, palpitations, dizziness, drowsiness or confusion, itching or tingling, pain and burning at the injection site, inflammation, injection abscess, bleeding or bruising, other).

4. Acute renal failure (urine output $<25 \mathrm{~mL} /$ hour) after initiation of treatment.

5. Pulmonary oedema.

6. Cerebrovascular accident (stroke).

7. Cardiac arrest.

8. Liver failure.

9. Coagulopathy.

10. Need for dialysis.

11. Need for ventilation.

12. Admission to ICU.
13. Length of stay (antepartum, postpartum total).

14. Time from admission to delivery.

15. Complications of delivery (placental abruption, retained placenta, postpartum haemorrhage).

16. Maternal death.

Neonatal:

1. Neonatal outcome at delivery (live birth vs stillbirth).

2. APGAR scores at 1 and $5 \mathrm{~min}$.

3. Need for NICU admission.

4. Reason for NICU admission.

5. Duration of NICU admission.

6. Neonatal outcome at discharge (alive vs dead).

\section{Statistical design}

Data will be entered into REDCap and analyses will be carried out using STATA. Primary study aim is to determine whether use of the 12 hours versus 24 hours modified Pritchard regimens of magnesium sulfate impact occurrence of seizure. First, bivariate analysis will be performed to determine whether there are baseline differences between conditions (12hours regimen, 24 hours regimen) in terms of demographics, obstetric history, medical history and index pregnancy. Comparisons across regimens will be performed using independent samples t-tests for normally distributed continuous variables, Wilcoxon rank test for non-normally distributed continuous variables and $\chi^{2}$ and Fisher's exact tests, where appropriate in the case of categorical variables. Next, the primary outcome of occurrence of a maternal seizure will be examined. Given the categorical nature of this outcome variable (yes seizure, no seizure), $\chi^{2}$ test of independence will be used to determine whether the probability of experiencing a seizure across conditions. Finally, analyses will be conducted to assess secondary maternal and neonatal outcomes. In the case of continuous outcome variables independent samples t-tests will be conducted to assess whether condition predicts the continuous secondary outcome of interest. In the case of categorical outcome variables, $\chi^{2}$ tests of independence will be conducted to determine whether the incidence of the categorical secondary outcome of interest differed by condition. For all analyses, Fischer's exact test will be used instead of a $\chi^{2}$ test of independence should the incidence of an outcome occur in less than five individuals per condition and (Bonferroni) corrections will be applied to correct for multiple comparisons. Number needed to treat and number needed to harm will be calculated for primary and secondary outcomes. All tests will be two-tailed. Of note, outcome data will be collected for participants who discontinue or deviate from intervention protocols.

\section{Data handling}

With approval from the IRB through the KBTH, Dr Titus Beyuo (primary author) will oversee the management of data collection and data safety at KBTH in Accra, Ghana.

Patients' data collection forms will be initially identified by their 'folder number' as given at their KBTH 
admission, as well as their name. This will allow subsequent data collection and integration throughout their hospital course, as well as data quality control as needed. Data collection forms will only be used and accessed by authorised study co-investigators and research assistants.

Data recorded on paper data collection forms will be reviewed by Dr Titus Beyuo and then entered into a secure electronic data organisation programme (REDCap) using a password protected computer. Only authorised study coinvestigators and research assistants will have access to this computer. The information entered into REDCap will only include unique study numbers and will not include participants names or any other personal identifying information.

The paper data collection forms will be stored securely in a locked cabinet in a locked office in the Obstetrics and Gynecology Department at KBTH, under the direction of Dr Titus Beyuo. The records (data collection sheets) will be retained for the entire duration of the study, during which data will be accessible only by authorised investigators. After complete cleaning and quality assurance, identification numbers will be replaced by simple sequence numbers. This de-identified data will be exported from REDCap into STATA for statistical analysis. All authors will have access to the final study dataset.

\section{Monitoring}

Monitoring for quality and regulatory compliance will be overseen by the KBTH Scientific and Technical Committee in Ghana. An independent Data and Patient Safety Board was established at KBTH, consisting of Professor K. Nkyekyer (consultant obstetrician and chairman), Professor K.A. Bugyei (clinical pharmacologist and member) and Dr Ayegua Hagan (statistician and member). The Data and Patient Safety Board was created to assess the progress of the clinical study, and will review cumulative study data to evaluate safety, study conduct and scientific validity and integrity of the study. Adverse events (anaphylaxis, allergic reaction to $\mathrm{MgSO}_{4}$, respiratory depression, coma) and severe adverse events (maternal morbidity or mortality secondary to anaphylaxis, allergic reaction to $\mathrm{MgSO}_{4}$, respiratory depression, coma) will be collected by the principal investigator, Dr Beyuo, and reported to the Data and Patient Safety Review Board. Dr Beyuo will also be responsible for communicating any adverse event or protocol modifications on behalf of the investigators to the Data and Patient Safety Board and the IRB.

\section{Termination of study}

The study may be terminated ahead of schedule on recommendation of the Data and Patient Safety Board or by the IRB or by the investigators if interim analysis (planned for 6 months after initiation of study) shows greater than $1 \%$ difference in recurrent fits between the study and control arms.

\section{Patient and public involvement statement}

This research was done without patient involvement. Patients were not invited to comment on the study design, interpret results or contribute to the writing or editing of this document.

\section{ETHICS AND DISSEMINATION}

Results of the study will be shared at clinical forums at the Korle Bu Teaching Hospital and will be submitted for publication in an international peer-reviewed journal.

Contributors TB and SAO were involved in conception of this research and initial trial design. All authors (TB, EL, ESL and SAO) were involved in review and finalisation of trial protocol. TB and EL were responsible for writing this paper, with review and editing from ESL and SO.

Funding This work was supported by the Ansbacher Award from the University of Michigan, through the Department of Obstetrics and Gynecology. The Ansbacher Award funders do not have a role in study design, data collection and analysis or decisions about where to submit for publication.

Competing interests None declared.

Patient consent for publication Not required.

Ethics approval Ethics approval was obtained in Ghana through the Korle Bu Teaching Hospital IRB. University of Michigan involvement is limited to protocol development and statistical analysis of de-identified data, and has been granted a Not Regulated Determination by the University of Michigan IRB. The study has been registered with the Pan African Clinical Trial Registry through the South African Medical Research Council: PACTR201811515303983.

Provenance and peer review Not commissioned; externally peer reviewed.

Open access This is an open access article distributed in accordance with the Creative Commons Attribution Non Commercial (CC BY-NC 4.0) license, which permits others to distribute, remix, adapt, build upon this work non-commercially, and license their derivative works on different terms, provided the original work is properly cited, appropriate credit is given, any changes made indicated, and the use is non-commercial. See: http://creativecommons.org/licenses/by-nc/4.0/.

ORCID iD

Emma Lawrence http://orcid.org/0000-0001-9606-7071

\section{REFERENCES}

1 Duley L. The global impact of pre-eclampsia and eclampsia. Semin Perinatol 2009;33:130-7.

2 WHO. Who recommendations for prevention and treatment of preeclampsia and eclampsia, 2011. Available: https://apps.who.int/iris/ bitstream/handle/10665/44703/9789241548335_eng.pdf;jsessionid= 476A1D103CE06844B2BD636E94D8E7DD?sequence $=1$

3 Adu-Bonsaffoh K, Oppong SA, Samuel OA, et al. Maternal deaths attributable to hypertensive disorders in a tertiary hospital in Ghana. Int J Gynaecol Obstet 2013;123:110-3.

4 Acquah-Arhin R, Kwawukuwe EY. Trends in eclampsia at Korle BU Teaching Hospatal, Accra Ghana. Niger J Clin Pract 2003;6:1-4.

5 Anjum S, Goel N, Sharma R, et al. Maternal outcomes after 12 hours and 24 hours of magnesium sulfate therapy for eclampsia. Int $J$ Gynaecol Obstet 2016;132:68-71.

6 Obed S, Aniteye P. Pregnancy following eclampsia: a longitudinal study at Korle-Bu Teaching Hospital. Ghana Med J 2007;41:139-43.

7 Okusanya BO, Oladapo OT, Long Q, et al. Clinical pharmacokinetic properties of magnesium sulphate in women with pre-eclampsia and eclampsia. BJOG 2016;123:356-66.

8 Fawcett WJ, Haxby EJ, Male DA. Magnesium: physiology and pharmacology. Br J Anaesth 1999;83:302-20.

9 Begum MR, Begum A, Quadir E. Loading dose versus standard regime of magnesium sulfate in the management of eclampsia: a randomized trial. J Obstet Gynaecol Res 2002;28:154-9.

10 Altman D, Carroli G, Duley L, et al. Do women with pre-eclampsia, and their babies, benefit from magnesium sulphate? The Magpie trial: a randomised placebo-controlled trial. Lancet 2002;359:1877-90. 
11 Ekele BA, Muhammed D, Bello LN, et al. Magnesium sulphate therapy in eclampsia: the Sokoto (ultra short) regimen. BMC Res Notes 2009;2:165.

12 Ehrenberg HM, Mercer BM. Abbreviated postpartum magnesium sulfate therapy for women with mild preeclampsia: a randomized controlled trial. Obstet Gynecol 2006;108:833-8.

13 Aali BS, Khazaeli P, Ghasemi F. Ionized and total magnesium concentration in patients with severe preeclampsia-eclampsia undergoing magnesium sulfate therapy. J Obstet Gynaecol Res 2007;33:138-43.
14 Chuan FS, Charles BG, Boyle RK, et al. Population pharmacokinetics of magnesium in preeclampsia. Am J Obstet Gynecol 2001;185:593-9.

15 Long Q, Oladapo OT, Leathersich S, et al. Clinical practice patterns on the use of magnesium sulphate for treatment of pre-eclampsia and eclampsia: a multi-country survey. BJOG 2017;124:1883-90.

16. Task Force on Hypertension in Pregnancy. Hypertension in pregnancy: Executive summary. Obstet Gynecol 2013;122.

17 Which anticonvulsant for women with eclampsia? Evidence from the collaborative eclampsia trial. Lancet 1995;345:1455-63. 